\title{
Análisis de una red colaborativa basado en el uso de un sistema informático que permite compartir auto en un ámbito universitario
}

\section{Analysis of a collaborative network based on the use of a computer system that allows auto sharing in a university context}

GONZÁLEZ-SILVA, Marco Antonio †*, HERNÁNDEZ-PÉREZ, Faride, BASURTO-FLORES, Rogelio y OLVERA-MEJÍA, Yair Félix

Universidad Politécnica Metropolitana de Hidalgo, Departamento de Ingeniería en Tecnologías de la Información

ID $1^{\mathrm{er}}$ Autor: Marco Antonio, González-Silva / ORC ID: 0000-0002-3327-8047, Research ID Thomson: U-8432-2018, CVU CONACYT ID: 173601

ID $1^{\mathrm{er}}$ Coautor: Faride, Hernández-Pérez / ORC ID: 0000-0001-9426-4944, CVU CONACYT ID: 557262

ID $2^{\text {do }}$ Coautor: Rogelio, Basurto-Flores / ORC ID: 0000-0003-4237-9074, CVU CONACYT ID: 368907

ID $3^{\text {er }}$ Coautor: Yair Félix, Olvera-Mejía / ORC ID: 0000-0003-4116-088X, Researcher ID Thomson: U-8258-2018, CVU CONACYT ID: 254526

DOI: $10.35429 /$ JTAE.2019.9.3.9.16

Recibido: 04 de Junio, 2019; Aceptado 30 de Septiembre, 2019

\begin{abstract}
Resumen
Un modelo tecnológico que ha tenido gran auge es la vinculación de personas que se da a través de grupos virtuales creados en medios digitales, también llamadas redes sociales. Este artículo presenta un análisis de una red social colaborativa cuyo diseño se basa en la estructura organizacional de una universidad. Por medio de implementar un sistema informático que busca promover la cultura de compartir automóvil, y así mejorar las condiciones de transporte de su comunidad, es posible encontrar relaciones simétricas y asimétricas que parten de las propias reglas de asociación de los usuarios en la universidad. Basado en este estudio se puede predecir el comportamiento de la red gracias a los patrones de conducta observados. Estas predicciones son de gran ayuda en la planeación de actividades a futuro donde se busca que la red tenga cierto comportamiento de colaboración entre sus individuos cuando se trata de realizar acciones con un beneficio común.
\end{abstract}

Análisis, Red Social, Colaborativa

\begin{abstract}
A technological model that has had great growth is the linking of people through virtual groups created in digital media, also called social networks. This article presents an analysis of a collaborative social network whose design is based on the organizational structure of a university. By means of implementing a computer system that promotes a service of car sharing, and thus improve the transport conditions of its community, it is possible to find symmetrical and asymmetric relationships that they come of common user association rules in the university. Based on this study, the behavior of the network can be predicted thanks to the observed behavior patterns of users. These predictions are of great help in the planning of future activities where the network is expected to have certain collaborative behavior among its individuals when it comes to performing actions with a common benefit and achieve goals planned in the future.
\end{abstract}

Analysis, Social Network, Collaborative

Citación: GONZÁLEZ-SILVA, Marco Antonio, HERNÁNDEZ-PÉREZ, Faride, BASURTO-FLORES, Rogelio y OLVERA-MEJÍA, Yair Félix. Análisis de una red colaborativa basado en el uso de un sistema informático que permite compartir auto en un ámbito universitario. Revista de Tecnología y Educación. 2019. 3-8: 9-16

\footnotetext{
* Correspondencia del Autor: (maagonzalez@upmh.edu.mx)

$\dagger$ Investigador contribuyendo como primer autor.
} 


\section{Introducción}

Una estructura compuesta por un conjunto de personas relacionadas entre sí, donde existe interacción entre ellas por pertenecer a una determinada contextualización de algún fenómeno o situación común, se conoce como red social (Kadushin, 2012). Actualmente, el análisis de las redes sociales ASN (por sus siglas en inglés Analysis of Social Networks) es considerado, por muchos autores, como una metodología de investigación donde se busca, entre otras cosas, encontrar los patrones de asociación de sus individuos. La utilidad de ASN ha sido enfocada a estudiar diversas variables de entes sociales bien identificados y delimitados como los habitantes de una determinada región, los empleados de una empresa o los estudiantes de una universidad, todo con el fin de entender porque se presentan ciertas conductas, tendencias 0 comportamientos que influyen positiva o negativamente en el desarrollo de dicho ente.

Encontrar dichos patrones de conducta ha permitido a los analistas saber, en muchos casos, las causas de cómo y por qué varían ciertos procesos como el crecimiento en la economía de una región, los índices de contaminación en algunas zonas geográficas y en distintos horarios, la migración, tendencias políticas, demanda de productos, etc. Este tipo de análisis de medición en las relaciones sociales existe desde los orígenes de la Sociometría, pero de acuerdo con la INSNA (International Network for Social Network Analysis), la tendencia de utilidad se orienta recientemente hacia diversos temas como salud, sociología e incluso estudio de la historia (Molina, 2009).

Uno de estos entes sociales, donde es posible aplicar el ASN, son los ambientes universitarios. De acuerdo al anuario estadístico de educación superior 2017-2018 emitido por la Asociación Nacional de Universidades e Instituciones de Educación Superior, la matrícula de estudiantes registrados en México es de 4,210,250 que equivale a un $2.7 \%$ más que el registrado en el periodo anterior (ANUIES, 2018). Esto se traduce a que ha habido un incremento de alumnos buscando hacer una carrera universitaria. Por lo tanto, es importante estudiar estos espacios donde la formación de personas es imprescindible en el desarrollo de la sociedad.
En las universidades, muchas de las relaciones entre sus integrantes se establecen por la misma estructura organizacional definida, esto es, personas que estudian la misma carrera, que pertenecen a la misma área de trabajo o que tienen el mismo jefe en común. Los objetivos que persigue una universidad suelen tener el común denominador de formación de recursos humanos con competencias profesionales, además del desarrollo de proyectos científicos y sociales, y es responsabilidad de la misma institución propiciar las condiciones de trabajo y colaboración para alcanzar las metas señaladas. En este escenario, es posible pensar que una red social pueda tener un rol fundamental para cumplir varios de estos objetivos, además su comportamiento puede contribuir a compartir conocimiento y crear grupos más fuertes de trabajo, que es la base de un aprendizaje colaborativo (Hinojo et al. 2018). Más aún, algunos autores señalan estudios donde se proponen modelos basados en redes sociales para mejorar el aprendizaje en los alumnos (AlRahmi et al. 2017).

En este artículo se muestra la estructura y análisis de la red social formada por la comunidad de la Universidad Politécnica Metropolitana de Hidalgo (UPMH). En ella se observan y estudian grupos definidos y que tienen poca comunicación entre ellos, esto según algunas reglas de asociación consideradas. Esta dispersión genera poca colaboración entre áreas o grupos que afectan diversos programas donde se requiere trabajo multidisciplinar.

\section{Hipótesis}

Por medio de un sistema informático, que permite la gestión de compartir auto entre miembros de la comunidad universitaria de la UPMH, se puede manipular el comportamiento de su red social mejorando las relaciones entre sus miembros. La hipótesis central en este trabajo es que el uso de este sistema pueda crear nuevas reglas de asociación que den como resultado una nueva estructura de red social colaborativa con menos centralización. 


\section{Objetivo}

El objetivo de este trabajo es analizar como la implementación y uso de un sistema informático, que promueve la cooperación entre miembros de una comunidad como la UPMH, permite crear nuevas reglas de asociación en la red social de esta entidad buscando un bien común y haciendo que ésta modifique su estructura.

Basada en una metodología mixta, cuantitativa y cualitativa se hizo una recopilación de datos sobre las principales relaciones que existen en la red social de la UPMH, donde se desea estudiar el número de vínculos y la calidad de ellos. Para una muestra representativa de la red se obtuvieron algunas métricas de centralización para conocer el grado de vinculación entre nodos que se traduce en nivel de colaboración entre ellos. La implementación del sistema informático de compartir auto se realizó con el fin de modificar el comportamiento de relación entre los individuos. Después de un periodo de prueba se analizaron nuevamente las métricas de centralización dando pie al surgimiento de nuevas reglas de asociación.

La estructura de este trabajo está organizada de la siguiente manera. En la sección 2 se presenta el trabajo relacionado con aquellos proyectos más relevantes donde se use al análisis de redes sociales para definir y predecir comportamientos. En la sección 3, marco teórico, se mencionan los conceptos básicos empleados para el análisis de la red social. En la sección 4, metodología, se hace mención de las técnicas y elementos utilizados en este proyecto. En la sección 5, desarrollo, se detallan los estudios, implementación y análisis realizados en el comportamiento de la red de la UPMH, antes y después de usar el sistema informático de compartir auto. En la sección 6 se presentan los resultados y conclusiones de este trabajo. En la sección 7, recomendaciones, se hace una breve proyección del trabajo a futuro. En la sección 9, referencias, se muestra la documentación consultada.

\section{Trabajo Relacionado}

El estudio de una estructura social, bajo el concepto de red y teoría de grafos, ha tenido gran relevancia en diversas áreas. A continuación se mencionan algunos ejemplos de su aplicabilidad.
En universidades y centros de investigación es importante conocer el perfil de aquellas personas que desempeñan labores científicas y predecir si su productividad será alta o no, y en cierta medida, determinar su rentabilidad. Masum et al. 2015, predijeron la productividad de miles de investigadores por crear una red social entre ellos y entrenar y utilizar un clasificador basado en máquinas de soporte vectorial (SVM).

En el ámbito de venta de productos, existen varios trabajos donde se analizan las redes sociales del mercado para predecir ingresos y demanda. En un trabajo propuesto por Hyung-1l 2014, se presenta un modelo predictivo de ventas de automóvil basado en series de tiempo y el análisis de la red social del consumidor donde las variables a estudiar son palabras clave que sean un común denominador y definan cierta tendencia de ventas. Otro trabajo que analiza las palabras o frases que se envían a través de las redes sociales es el presentado por D’Avanzo et al 2017. En este trabajo se hace un estudio de las palabras que se ingresan en buscadores como google para determinar tendencias y emociones de una cierta población, y no solo dejar estos datos como simples estadísticas de búsqueda.

En otro campo de aplicación de ASN se pueden encontrar investigaciones para predecir futuras enfermedades. Por ejemplo, en el trabajo propuesto por Kaya y Poyraz 2015, se construyó una red de enfermedades ponderadas y luego un método de predicción de relaciones entre ellas para entender cómo es que evolucionan en los pacientes.

\section{Marco Teórico}

\section{Principios}

\section{Grado}

El grado de un nodo en una red es el número de conexiones asociadas a cada vértice. Si una red es dirigida, esto significa que habrá enlaces que salgan y otros que entren de los nodos y por lo tanto se mencionará que existen grados de salida y de entrada en función de la disposición de los enlaces entrantes y salientes de los nodos. (Erik McCullough, 2005) 


\section{Distribución de grado}

Es un función de probabilidad que indica en que porcentaje un nodo puede tener un determinado grado. Si se hiciera un recuento en una red del número de nodos por cada grado se tendría una distribución de grado que sería entendido igualmente como la distribución de probabilidad de un grado en la red. (AlbertLászló Barabási, 2003). En redes libres de escala, como redes sociales, la probabilidad de tener un grado alto recae solo en algunos nodos.

\section{Herramientas}

\section{Python}

Se trata de un lenguaje de programación multiparadigma que soporta orientación a objetos, programación imperativa y, en menor medida, programación funcional. Es un lenguaje interpretado, dinámico y multiplataforma. (Knowlton, Jim, 2009).

\section{NetworkX}

NetworkX es una paquetería echa en Python para la creación, manipulación y estudio de estructuras dinámicas y redes complejas

\section{Gephi}

Gephi Es software open-source de análisis de redes y visualización escrito en Java en la plataforma NetBeans.( Bastian, Mathieu, 2009)

\section{Metodología}

La teoría de redes complejas es una de las nuevas áreas de la ciencia que está interesada en la forma en que se organizan sus elementos. Su uso se ha extendido a lo largo de diferentes campos de estudio, si bien nació en los laboratorios de las notas de los matemáticos como Erdös y Renyi, ahora se le puede observar en los estudios de relaciones sociales, de economía en la forma en que se llevan a cabo los negocios internacionales, en el diseño de medicamentos para ingeniería metabólica, en la detección temprana de sismos y en redes neuronales (Reka, 2002, Aguilar et al., 2019, Sporns et al., 2005, Aguilar et al., 2013 y Goh et al., 2007).
Para describir la metodología de este trabajo se considera importante definir los siguientes conceptos. Se puede considerar una red compleja a través del conjunto de nodos $N$, y las relaciones entre dichos nodos denotadas mediante otro conjunto de elementos llamados vértices, $V$. La complejidad de la red siempre estará dada por el número de elementos que tiene y la relación de los mismos. Existen muchos tipos de redes, para poder caracterizarlas se han diseñados métricas específicas para los distintos problemas en que son utilizadas. Algunas métricas de importancia son la distribución de grado, el coeficiente de agrupamiento (clustering), la modularidad, y su centralidad.

El grado de un nodo se calcula con los enlaces que tiene asociados, de esta forma también, es posible calcular su distribución al contar el número de nodos con determinado grado. Dicho conteo puede categorizar las redes en aleatorias y libres de escala. Las redes aleatorias se caracterizan por una distribución de grado normal, las libres de escala, como su nombre lo dice no tienen una escala determinada; dado que, al calcularse la distribución de grado, el resultado es una ley de potencias, la forma de caracterizarlas es con la pendiente que se obtiene de generar una gráfica $\log -\log$ de dicha ley de potencias (Newman, 2010, Erdös et al., 1959).

La idea de centralidad de una red se basa en medir la importancia de un elemento en ella, ya sea un nodo o un enlace. La forma de calcular la centralidad se puede hacer de varias formas, como el grado máximo en una red (centralidad de grado), o bien, el número de veces que un camino más corto entre dos nodos pasa por un vértice (la centralidad de intermediación o betweenness centrality).

Este tipo de centralidad necesita del concepto de camino, mismo que se define como la secuencia de vértices, de tal forma que cada par de vértices consecutivos están conectados a través de un mismo nodo. El camino más corto se calcula viendo todos los posibles caminos entre un par de nodos dado y obteniendo aquel que tenga el menor número de vértices (Nicosia et al., 2011).

De tal forma que, para obtener la centralidad para cada nodo $i$, llamada $g(i)$, se calcula lo siguiente, 
$g(i)=\sum_{s \neq i \neq t} \frac{\sigma_{s t}(i)}{\sigma_{s t}}$

donde $\sigma_{\mathrm{st}}$ es el número total de caminos más cortos desde el nodo $s$ hasta el nodo $t$, y $\sigma_{\mathrm{st}}(\mathrm{i})$ es el conjunto de los caminos más cortos que pasan por el nodo $i$. Normalizando los valores por el número de pares de nodos existentes en la red, de tal forma que los resultados por nodo son entre 0 y 1 . En este artículo se hace un análisis de centralidad de red usando el coeficiente de agrupamiento presentado en la fórmula (1).

\section{Desarrollo}

\section{Investigación previa}

Se realizó una investigación del comportamiento actual de la red social de la UPMH. Para esto se tomó una muestra de 280 alumnos, y se aplicó una encuesta para conocer su entorno social, esto es, ¿qué alumnos tienen una comunicación entre ellos sin que sea por una razón académica?. Para procesar los datos y generar el grafo correspondiente, se utilizó el lenguaje de programación Python, en específico con la librería NetworkX. Esta librería es un paquete para la creación, manipulación y estudio de la estructura, dinámica y funciones de redes complejas. Adicionalmente, se utilizó el software gephi, que es una herramienta opensource desarrollada en Java para visualizar y analizar grandes gráficos de red.

Utilizando las herramientas antes mencionadas y los datos arrojados por la encuesta, se obtuvo el grafo de la figura 1.

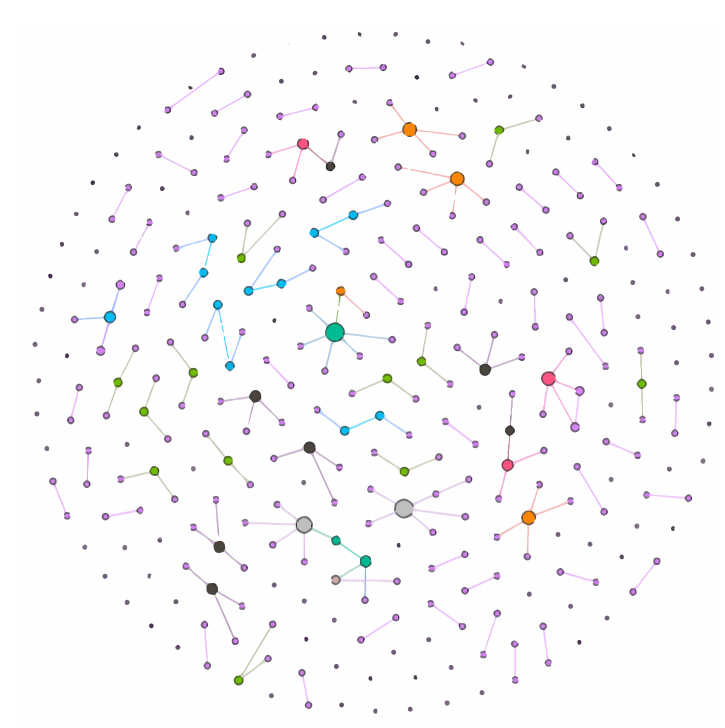

Figura 1 Red de relaciones de amistad entre estudiantes
Como se puede observar en la Figura 1, los nodos, que representan a los estudiantes, tienen un grado de centralidad muy bajo, siendo 5 el valor máximo de la red, esto indica que estos nodos se comunican poco entre ellos.

Otro de los datos que se obtuvo de la encuesta, es la relación obligada que tienen los estudiantes por pertenecer a una misma carrera, generando el grafo de la figura 2.

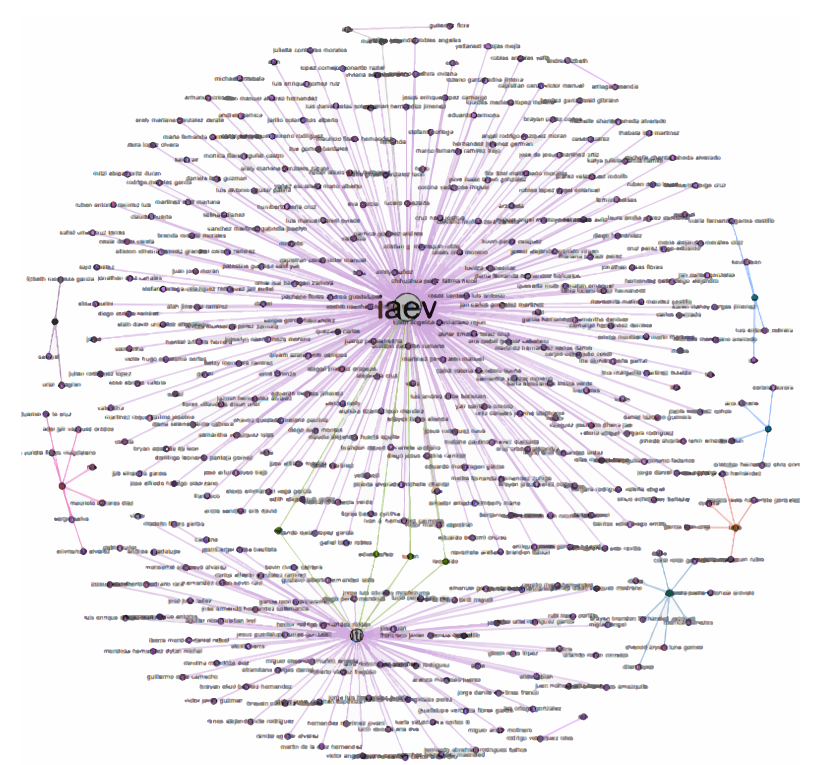

Figura 2 Red de relaciones entre estudiantes a través de su carrera

Como se puede observar en la Figura 2 la encuesta revela que el entorno social de los estudiantes está completamente dirigido por la carrera en la que se encuentran estudiando, lo cual limita muchas oportunidades de crecimiento para estudiantes y para la UPMH, como por ejemplo el desarrollo de proyectos colaborativos.

\section{Implementación Lion Way}

Al observar el comportamiento de la red social dentro de la comunidad de la UPMH (figuras 1 y 2), e identificar los problemas que conlleva tener baja comunicación entre nodos, se desarrolló e implemento la aplicación LionWay. LionWay es un sistema para compartir auto dentro de la comunidad universitaria (UPMH), incluyendo estudiantes, maestros y personal administrativo. El objetivo de ésta es modificar la red social de la UPMH, buscando crear mayor colaboración entre nodos. Para ello, este sistema se diseñó de tal modo que obliga a la comunidad a compartir auto con personas de carreras o puestos diferentes a los que pertenecen. 


\begin{abstract}
Lionway funciona de la siguiente manera. Existe un portal web (www.lionway.xyz) donde es posible crear un perfil y especificar datos personales y ubicación de cada usuario. Al ingresar un usuario puede publicar un viaje, es decir, un conductor de un auto puede divulgar información de un recorrido que va a realizar especificando el punto de partida, la ruta que seguirá y los lugares disponibles dentro de su vehículo. A su vez, un pasajero podrá ver las publicaciones y apartar un lugar en el auto de alguna publicación de viaje. De esta manera, utilizando la necesidad de transportase de manera eficiente de la comunidad universitaria se manipula el comportamiento de comunicación de la red social de la UPMH, generando nuevos vínculos dentro de la red, siendo estos no dependientes de la carrera a la cual una persona pertenece.
\end{abstract}

\section{Resultados}

La aplicación LionWay estuvo en funcionamiento 3 meses en la comunidad universitaria, tomando la misma muestra de 280 alumnos citada en la sección 5.1.

Al finalizar el periodo de prueba, se implementó de nuevo la encuesta, obtenido los resultados de la figura 3 .

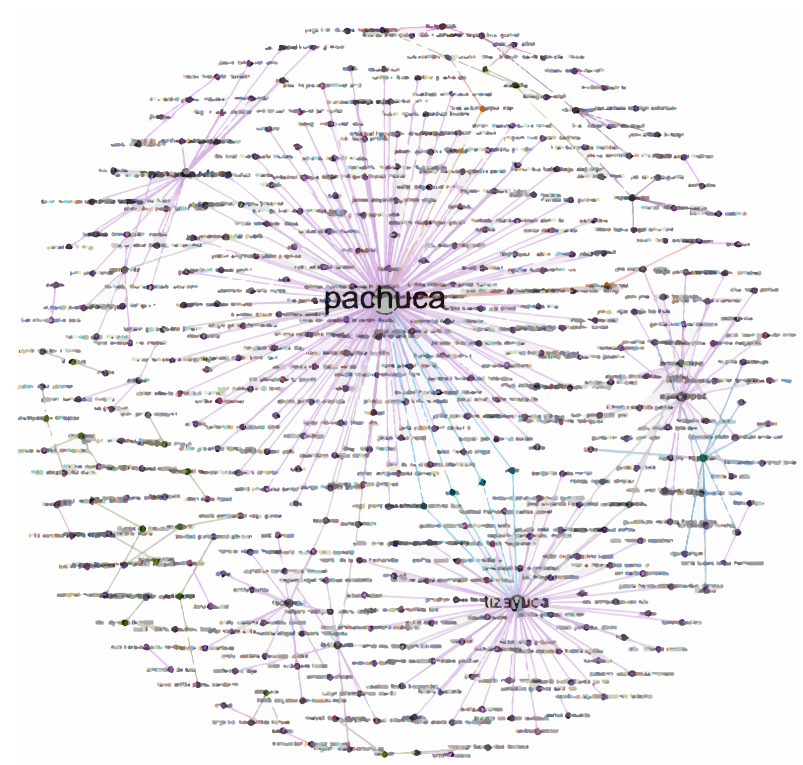

Figura 3 Red de relaciones entre estudiantes a través de su lugar de residencia

Como se puede observar en la Figura 3, la red ha cambiado notablemente su nivel de agrupamiento. Se han creado nuevas relaciones, generando nodos con nivel de grado mayor, y provocando aglomeramiento dentro de la red, fortaleciendo las relaciones dentro de ella.
Como producto de las mediciones de las relaciones entre estudiantes a través de los lugares de residencia obtenidos de la aplicación, se puede observar que las relaciones cambian y se generan más grupos; el grupo central, el de la ciudad de Pachuca el más grande.

En la Figura 2 se tiene una centralidad máxima de 0.6, mientras que en la Figura 3 se tiene una centralidad de 0.55 , haciendo que los nodos centrales sean menos importantes en la Figura 3, debido a la diversificación de destinos. Si bien la diferencia no es mucha se puede observar mejor cuando se compara la cantidad de elementos menos centrales entre ambos grafos: $65 \%$ en la primera y $35 \%$ en la segunda.

En la figura 4 se muestran los datos de distribución de grado de los nodos después de la aplicación de LionWay.

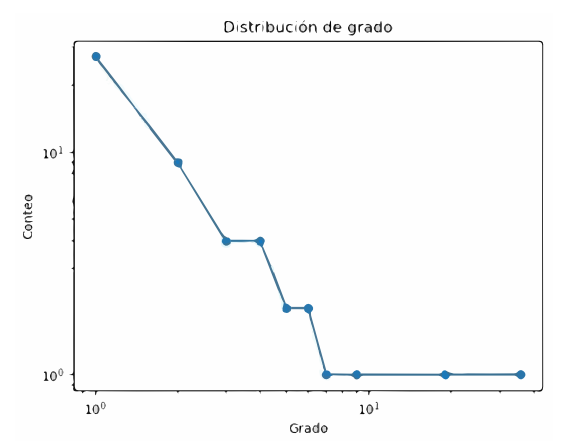

Figura 4 Distribución de grado

En la Figura 4 se puede observar la relación entre nodos y el nivel de grado que tienen, con ella se puede concluir que la red se va modificando paulatinamente en su comportamiento, generando que los nodos aumenten de grado, y comenzando a cambiar la forma de una red libre de escala donde solo unos cuantos nodos tienen grados altos.

Con esto se demuestra que la implementación de LionWay generó un cambio en el entorno social de la comunidad Universitaria. Como trabajo a futuro se pretende analizar la red con un periodo de prueba más largo, aplicando diferentes tipos de métricas para conocer los cambios de la red bajo otro tipo de enfoque. 


\section{Conclusiones}

Con los resultados obtenidos se puede concluir que el uso de herramientas informáticas, que requieren de la participación de la mayoría de los miembros de una comunidad, se pode modificar el comportamiento de una red social. Esto puede orientarse para lograr objetivos específicos como trabajos interdisciplinarios o colaborativos que buscan un bien común entre sus miembros.

\section{Recomendaciones}

En proyectos donde se busca analizar el comportamiento de redes sociales, donde existe un gran número de miembros en ellas, es conveniente plantear pequeños grupos de estudio donde los resultados se puedan medir y analizar, incluso, en elementos individuales. Estos elementos en cierta medida son un punto de referencia para comprobar hasta que grado se puede manipular el comportamiento de la red $\mathrm{y}$ de sus miembros con herramientas informáticas externas, como la aquí expuesta, que busca generar cambios de comportamiento.

\section{Agradecimientos}

Al programa PRODEP por el apoyo prestado al proyecto "Monitoreo de Variables Continuas y Discretas vía Aplicaciones Móviles para Automatización de Procesos" desarrollado por el cuerpo académico "Sistemas y Tecnologías de la Información" con clave UPMDH-CA-7

\section{Referencias}

Aguilar S.J.B. y Guzmán V.L. Earthquake magnitude time series: scaling behaviour of visibility networks. The European Physical Journal B. Vol. 92 (424). Noviembre 2013.

Aguilar V.D. y Guzmán V. L. (2019). Critical Synchronization and $1 / \mathrm{f}$ noise in inhibitory/excitatory rich-club neural networks. Nature International Journal of Science, Scientific Report No. 9, 2019, pp. 1-13.

Albert-László Barabási, (2003) "Linked: The New Science of Networks", Basic Books, ISBN 0-7382-0667-9
Al-Rahmi W. M. y Zeki A. M. "A model of using social media for collaborative learning to enhance learners's performance on learning". Journal of King Saud University - Computer and Information Sciences, Vol. 29, Octubre 2017, pp. 427-562.

ANUIES, 2018. Anuario de Educación Superior Licenciatura 2017-2018. http://www.anuies.mx/informacion-yservicios/informacion-estadistica-de-educacionsuperior/anuario-estadistico-de-educacionsuperior, consultado 20-mayo 2018, línea 4210250.

Bastian, Mathieu; Heymann, Sebastien; Jacomy, Mathieu (2009), Gephi : An Open Source Software for Exploring and Manipulating Networks, AAAI Publications, Third International AAAI Conference on Weblogs and Social Media, consultado el 22 de noviembre de 2011

D’Avanzo E., Pilato G. y Lytras M. (2017). Using Twitter Sentiment and Emotions Analysis of Google Trend for Decisions Making. Programa de librería electrónica y sistemas de información. Universidad de Belfast. Julio 2017

Erdös P. y Rényi, A. (1959). On random graphs, i. Publicationes Mathematics (Debrecen), 6, 290-297.

Erik McCullough, (2005) "Random Networks with Tunable Degree Distribution and Clustering", Volz, Cornell University, Jan.

Go K., Cusick M., Valle D., Childs B., Vidal M. y Barabási A. (2007). The Human Disease Network. The National Academy of Sciences of the USA.Vol. 104 No. 21. Mayo 2007. pp. 8685-8690

Hinojo L. F., Aznar D.I., Cáceres R. M., y Romero J. M. (2018). "Use of Social Network for International Collaboration among Medical Students". Revista de Educación Médica, El Sevier, No.415, Agosto 2018, pp. 1-5.

Hyung-ll A. y Spangler S.W. (2014). Sales Prediction with Social Media Analysis. Conferencia Anual SRII IEEE, San José, California, Estados Unidos, Agosto 2014. 
Kadushin Ch. (2012). Comprender las redes sociales, teorías, conceptos y hallazgos. Centro de Investigaciones Sociológicas Oxford, 1ra Ed. Madrid, Esp. 2012 pp. 335.

Knowlton, Jim (2009). Python. tr: Fernández Vélez, María Jesús (1 edición). Anaya Multimedia-Anaya Interactiva. ISBN 978-84415-2513-9.

Masum S.B. y Gauch S. (2015). Social Network Analysis for Predicting Emerging Researches. 7a. Conferencia Internacional sobre Descubrimiento del Conocimiento y Recuperación de la Información. Lisboa, Portugal, Noviembre 2015

Molina J.L. (2009). Panorama de la Investigación en Redes Sociales. Redes. Revista Hispana para el Análisis de Redes Sociales, vol. 17, diciembre 2009, pp. 235-256

Newman M. (2010). Networks An Introduction. Oxford University Press. New York, Estados Unidos 2010, pp. 720

Nicosia V, Criado R., Romance M., Russo G. y Lotara V. 2011. Controlling Centrality in Complex Networks. Reporte Física y Sociedad, Cornel University, New York, USA. pp 1-7.

Reka A. y Barabasi A. (2002). Statistical Mechanics of Complex Networks. Reviews of Modern Physics. University of Notre Dame. Vol. 74. 2002. pp. 47-97

Sporns O., Tononi J. y Köter R. (2005). The Human Connectome: A structural Description of the Human Brain. Plos Computational Biology. Vol.1 (4). Septiembre 2005. pp. 245251.

Watts D. y Strogatz S.H. (1998). Collective Dynamics of small world networks. Nature Vol. 393. Junio 1998. pp. 440-442. 